\title{
Essential fatty acids and mental health
}

\author{
BRIAN HALLAHAN and MALCOLM R. GARLAND
}

The biological basis for the major psychiatric disorders is presumed to be a deficit or excess of neurotransmitters or abnormalities in their interactions with their respective receptors or transporters. Accordingly, the vast bulk of biological research, from genetics to psychopharmacology and from the study of signal transduction systems to in vivo molecular imaging, has placed the neurotransmitter and its target proteins centre-stage. This belies the fact that the dry weight of the mammalian brain is approximately $80 \%$ lipid (the highest of any organ) and also the steady accumulation of data demonstrating the crucial role of lipids, particularly long-chain polyunsaturated fatty acids (LC-PUFAs), in modulating neural function. The essential fatty acids (EFAs) are LC-PUFAs obtained exclusively through diet and they comprise $15-30 \%$ of the brain's dry weight. Their effect on neuronal membrane dynamics and therefore on receptor, transporter and neurotransmitter function is profound (see below). Moreover, the well-documented shift in the Western diet away from EFAs (and the omega-3 family in particular) parallels the large rise in all psychiatric disorders seen over the past century. Finally, along with the resurgence of interest in lipid-neuronal membrane interactions, there are now a considerable number of quality randomised controlled trials demonstrating the efficacy of EFAs in a diverse number of psychiatric conditions. (Additional references to those in the reference list are available from the corresponding author on request.)

\section{FUNCTIONAL BIOCHEMISTRY OF THE ESSENTIAL FATTYACIDS}

The EFAs are divided into two groups, omega-3 $(\omega-3)$ and omega-6 ( $\omega-6)$ fatty acids, depending on where the first double bond in the carbon chain occurs. The principal sources of EFAs are oily fish and certain vegetable oils. These are then processed into other long-chain polyunsaturated fatty acids (LC-PUFAs) by elongation and desaturation. The principal central nervous system-related EFAs are eicosapentaenoic (EPA) and docosahexaenoic (DHA) acids (both $\omega-3$ ) and arachidonic acid ( $\omega-6)$. They are important components of phospholipids and cholesterol esters, which are themselves integral to the neuronal cell membrane, especially synaptic and dendritic membranes, but also intracellular membranes such as mitochondria and vesicles. If unavailable, they will be replaced by non-EFAs, changing the behaviour of the phospholipid molecules and affecting the tertiary and quaternary structures of membranebound receptors and associated neurotransmitters. The membrane phospholipid bilayer forms the matrix within which membrane proteins, such as receptors and ion channels, are embedded and to which membrane-associated proteins involved in second messenger systems are attached.

Additionally, cell signalling systems are regulated by lipid products, such as diacylglycerols, prostaglandins, leukotrienes, free fatty acids and hydroxy-fatty acids derived from phospholipid membranes. Moreover, EFAs are the precursors of the eicosanoids, a complex group of highly biologically active compounds encompassing the prostanoids (including prostaglandins, thromboxanes and prostacyclins) and leukotrienes. Eicosanoids derived from $\omega-3$ EFAs have fewer inflammatory effects than those derived from $\omega-6$ EFAs, underpinning the opposing effects that the $\omega-6$ EFA families sometimes have (see below). Eicosanoids perform numerous regulatory functions in the brain and throughout the rest of the body, in particular for the regulation of immune and inflammatory responses.
There are now numerous animal studies relating to a wide variety of adverse effects associated with diets low in EFAs. As a converse example, De la Pressa Owens \& Innis (1999) demonstrated that in pigs supplemented with EFAs from birth there was a doubling of the major monoamines in the frontal cortex.

\section{MODERN DIET \\ IS ASSOCIATED WITH DRAMATIC REDUCTIONS IN EFA INTAKE}

The modern Western diet has evolved into a meat and saturated fat-rich diet, with falling consumption of fresh vegetables and fish. This has been coupled with a staggering rise in the consumption of seed oils (such as sunflower and soybean), whose polyunsaturated fatty acid content is predominantly $\omega-6$, at the expense of $\omega$ 3 , EFAs. The $\omega-6: \omega-3$ ratio is estimated to have been 0.4-2.8:1 in Palaeolithic and evolutionary diets (Eaton et al, 1998). These models did not even consider seafood consumption, which would bring the ratios even lower. The $\omega-6: \omega-3$ ratio is now estimated to have risen to $17: 1$ in our typical Western diet (Eaton et al, 1998). Moreover, wild and free-ranging animals have significantly more $\omega-3$ fatty acids in their tissues than do currently produced commercial livestock. An overproduction of pro-thrombotic eicosanoids such as thromboxane, as well as pro-inflammatory leukotrienes and cytokines from a diet over-rich in $\omega-6$ EFAs, has resulted and may be contributing to the rise in the incidence of such diseases as atheroma, thrombosis and a variety of inflammatory conditions (Pischon et al, 2003). Such a sustained change in diet, as will now be discussed, may also be affecting mental health.

\section{DIETARY REDUCTIONS IN EFA PAR ALLELTHE RISE IN PSYCHIATRIC DISORDER}

Bearing in mind that fish are the principal source of $\omega$-3 EFAs, Hibbeln (1998) reports that the documented 60 -fold variation across countries in the annual prevalence of major depression is strongly inversely correlated with national fish consumption. A similar inverse relationship 
exists for the prevalence of post-partum depression and fish consumption. In a survey of 3204 adults in Finland, infrequent fish consumption was associated with depressive symptoms in women $(P<0.01)$ and a similar trend, although not statistically significant, was noted in men (Tanskanen et al, 2001).

For bipolar affective disorder, Hibbeln's group have demonstrated that prevalence rates rise precipitously below an apparent annual fish intake threshold of approximately $75 \mathrm{lb}$ per person, with prevalence rates of, for example, $0.04 \%$ in Taiwan (81.6 lb per person) and $6.5 \%$ in Germany (27.6 lb per person), which is a nearly 60 fold difference. The same group did not find a relationship between fish consumption and prevalence rates for schizophrenia.

For suicide, daily fish eating had a positive effect (odds ratio $=0.81$ ) in reducing the risk of death from suicide compared with subjects having a non-daily consumption in 17-year follow-up of 256118 Japanese subjects (Hirayama, 1990). With regard to dementia, in a study of elderly men, estimates of high $\omega-6$ fatty acid intake were associated with cognitive impairment and, conversely, fish consumption was inversely associated with cognitive impairment, the significance in both cases persisting after controlling for confounders (Kalmijn et al, 1997).

\section{LOWER EFA LEVELS IN PSYCHIATRIC ILLNESS}

In one of several consistent studies on depression, Peet et al (1998) demonstrated that total $\omega-3$ fatty acid levels were decreased $(5.39 \mathrm{mg} / 100 \mathrm{mg}$ phospholipid compared with $9.04 \mathrm{mg} / 100 \mathrm{mg}$ phospholipid for controls; $P=0.02$ ), DHA especially $(1.61 \mathrm{mg} / 100 \mathrm{mg}$ phospholipid compared with $2.50 \mathrm{mg} / 100 \mathrm{mg}$ phospholipid; $P=0.04$ ), on red blood cell membranes in patients with major depressive disorder $(n=15)$ compared with normal controls. The same group reported similar data in schizophrenia (Peet et al, 1995). We have demonstrated lowered plasma levels of cholesterol (Garland et al, 2000) and EFA (details available from the author upon request) in populations of patients with self-harm. There are now also considerable data supporting plasma EFA deficiency in attention-deficit hyperactivity disorder (e.g. Richardson \& Puri, 2000) and dementia (Tully et al, 2003).

BRIAN HALLAHAN, MRCPsych, MALCOLM R. GARLAND, MRCPsych, Royal College of Surgeons of Ireland and the Department of Psychiatry, Beaumont Hospital, Dublin, Ireland, UK

Correspondence: Malcolm R. Garland, Royal College of Surgeons of Ireland and the Department of Psychiatry, Beaumont Hospital, Dublin 9, Ireland, UK. Tel: 00353 I 8093354; fax: 00353 I 8376982; e-mail: mgarland@ireland.com

(First received 29 March 2004; final revision 14 September 2004, accepted 30 September 2004)

\section{SUPPLEMENTATION IN CLINICAL POPULATIONS: THE EVIDENCE}

All but one of the four EFA supplementation randomised controlled trials (RCTs) in depression report significant improvement with supplementation. The largest of these studies (Peet \& Horrobin, 2001) involved 70 patients, who remained on adequate standard therapy throughout the trial; at optimum dosage, there was a threefold treatment-to-placebo effect, using a $50 \%$ reduction in Hamilton Rating Scale for Depression (HRSD) scores as evidence of clinical response. For bipolar affective disorder, in a 4-month RCT (Stoll et al, 1999) of high-dose $\omega-3$ fatty acids, patients $(n=30)$ had longer periods of remission in the $\omega$-3-treated group $(P<0.02)$ and significant improvements in depressive, but not manic, symptomatology were observed, suggesting that depressive symptoms may be more responsive than manic symptoms to supplementation. For schizophrenia, the evidence is less equivocal. As reviewed recently (Joy et al, 2003), only one of five small studies demonstrated substantial clinical improvement, and larger studies are clearly needed. There are only limited and inconsistent data on supplementation in attention-deficit hyperactivity disorder and dementia, although there are striking data on Huntington's chorea ( $n=17$ patients) where treatment was associated not just with stabilisation but with reversal of clinical signs (Vaddadi et al, 2002).

There are various, generally positive data in psychopathic/aggressive/impulsive populations. In a carefully conducted RCT in young adult prisoners, a combination of $\omega-6$ and $\omega-3$ EFAs reduced offences by $26.3 \%$, increasing to $35.1 \%$ if on supplementation for a minimum of 2 weeks $(P<0.01 ;$ Gesch et al, 2002). The greatest reduction occurred for the most serious incidents including violence. As reviewed elsewhere (Hallahan \& Garland, 2004), EFAs have proved beneficial also in stabilising aggression in a normal population of young university students at exam time $(n=41)$ compared with randomised controls and in a population $(n=30)$ of patients with borderline personality disorder treated for 8 weeks. Finally, we are shortly to conclude recruitment into an RCT of EFAs in a population of patients with self-harm.

\section{CONCLUSIONS}

There is no doubt that cerebral lipids, and EFA-derived LC-PUFAs in particular, have significant direct and indirect actions on cerebral function. Not only does the lipid composition of neural membranes affect the function of their embedded proteins, but also many LC-PUFAs are converted to neurally active substances. There is good evidence that psychiatric illness is associated with depletion of EFAs and, crucially, that supplementation can result in clinical amelioration. As well as challenging traditional views of aetiology and therapeutics in psychiatry, the clinical trial data may herald a simple, safe and effective adjunct to our standard treatments for many disabling conditions.

\section{DECLARATION OF INTEREST}

None.

\section{REFERENCES}

De la Pressa Owens, S. \& Innis, S. M. (1999) Docosahexanoic and arachidonic acid prevent decrease in dopaminergic and serotonergic neurotransmitters in frontal cortex caused by a linoleic and alpha-linoleic acid deficient diet in formula-fed piglets. Journal of Nutrition, I29, 2088-2093.

Eaton, S. B., Eaton, S. B. 3rd, Sinclair, A., et al (1998) Dietary intake of long chain fatty acids during the palaeolithic. The return of omega-3 fatty acids to the food supply. I: Land-based animal products and their health effects. World Review of Nutrition and Dietetics, 83 , $12-23$.

Garland, M., Hickey, D., Corvin, A., et al (2000) Tota serum cholesterol in relation to psychological parameters in parasuicide. British Journal of Psychiatry, I77, 77-83.

Gesch, C. B., Hammond, S. M., Hampson, S. E., et a (2002) Influence of supplementary vitamins, minerals and essential fatty acids on the antisocial behaviour of young adult prisoners. British Journal of Psychiatry, 18I, 22-28. 
Hallahan, B. \& Garland, M. R. (2004) Essential fatty acids and their role in the treatment of impulsivity disorders. Prostaglandins, Leukotrienes and Essential Fatty Acids, 7I, 211-216

Hamilton, M. (1960) A rating scale for depression. Journal of Neurology, Neurosurgery and Psychiatry, 23, $56-62$

Hibbeln, J. R. (1998) Fish consumption and major depression. Lancet, 35I, 1213.

Hirayama, T. (1990) Life Style and Mortality. A Large Census-Based Cohort Study in Japan. Basel: Karger.

Joy, C. B., Mumby-Croft, R. \& Joy, L. A. (2003)

Polyunsaturated fatty acid supplementation for

schizophrenia. Cochrane Database Systematic Reviews, 2 CD00I257.

Kalmijn, S., Feskens, E. J., Launer, L. J., et al (1997) Polyunsaturated fatty acids, antioxidants, and cognitive function in very old men. American Journal of Epidemiology, 145, 33-41.
Peet, M. \& Horrobin, D. F. (200I) A dose-ranging study of ethyl-eicosapentonate in treatment-

unresponsive depression. Journal of Psychopharmacology, I5 (suppl.), Al2.

Peet, M., Laugharne, J., Rangarajan, N., et al (1995) Depleted red cell membrane essential fatty acids in drugtreated schizophrenic patients. Journal of Psychiatric Research, 29, 227-232.

Peet, M., Murphy, B., Shay, J., et al (1998) Depletion of omega-3 fatty acid levels in red blood cell membranes of depressive patients. Biological Psychiatry, 43, 315-319.

Pischon, T., Hankinson, S., Hotamisligil G. S., et al (2003) Habitual dietary intake of $n-3$ and $n-6$ fatty acids in relation to inflammatory markers among US men and women. Circulation, 108, 155-160.

Richardson, A. J. \& Puri, B. K. (2000) The potential role of fatty acids in attention-deficit/hyperactivity disorder. Prostaglandins, Leukotrienes and Essential Fatty Acids, 63, 79-87.
Stoll, A. L., Severus, E., Freeman, M. P., et al (1999) Omega-3 fatty acids in bipolar disorder. Archives of General Psychiatry, 56, 407-412.

Tanskanen, A., Hibbeln, J. R., Hintikka, J., et al (200I) Fish consumption and depression and suicidality in a general population. Archives of General Psychiatry, 58, 512-513.

Tully, A. M., Roche, H. M., Doyle, R., et al (2003) Low serum cholesteryl ester-docosahexaenoic acid levels in Alzheimer's disease: a case-control study. British Journal of Nutrition, 89, 483-489.

Vaddadi, K. S., Soosai, E., Chiu, E., et al (2002) A randomised, placebo-controlled, double blind study of treatment of Huntington's disease with unsaturated fatty acids. Neuroreport, 13, 29-33. 